Original Paper http://ajol.info/index.php/ijbcs http://indexmedicus.afro.who.int

\title{
Un vaccin bivalent efficace contre la maladie de Newcastle et la variole aviaire, produit au Sénégal
}

\author{
Ndeye Fatou Tall NDIAYE ${ }^{1 *}$, El Hadji TRAORE ${ }^{2}$ Mariane AIDARA $^{1}$

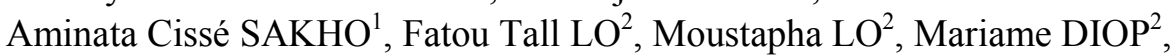 \\ Aminata Niang NDOYE ${ }^{2}$ et Abdoulaye DIENG ${ }^{3}$ \\ ${ }^{I}$ ISRA-Unité de production de vaccins, Sénégal. \\ ${ }^{2}$ ISRA-Laboratoire National de l'Elevage et de Recherches Vétérinaires, Sénégal. \\ ${ }^{3}$ Université de Thiès-Ecole Nationale Supérieure d'Agriculture, Sénégal. \\ *Auteur correspondant ; E-mail:ndeyefatou@hotmail.com
}

\section{REMERCIEMENTS}

Nous remercions le Fonds National pour la Recherche Agro-alimentaire (FNRAA), qui a financé ces travaux.

\section{RESUME}

La maladie de Newcastle (MN) et la variole aviaire sont des pathologies majeures de l'aviculture villageoise au Sénégal. Elles affectent la plupart des espèces d'oiseaux et causent des pertes économiques considérables dans les élevages. La vaccination est le seul moyen de prévention efficace ; d'où l'idée de mettre au point un vaccin bivalent pour lutter contre ces deux maladies. Le vaccin bivalent produit est constitué de la souche virale I-2 de la MN et d'une souche locale du virus de la variole aviaire (PGPV19). Pour évaluer l'efficacité du vaccin bivalent au laboratoire, des essais vaccinaux ont été réalisés sur des poulets âgés de 21 jours. La technique de l'inhibition de hémagglutination (IHA) a été utilisée pour déceler les anticorps contre la MN, tandis que le pouvoir immunogène de la souche PGPV19 était mesuré en éprouvant les sujets avec la souche sauvage S20 du virus de la variole aviaire. Les résultats obtenus sont concluants car les sujets vaccinés ont produit des anticorps neutralisants contre la $\mathrm{MN}$ et ont affiché une immunité lorsqu'ils ont été en contact avec la souche S20 contrairement aux sujets témoins. Toutefois, ces résultats, bien qu'encourageants doivent être confortés par des données comparatives issues de suivis d'élevages villageois.

(C) 2017 International Formulae Group. All rights reserved.

Mots clés : Aviculture villageoise, maladie de Newcastle, variole aviaire, vaccin bivalent, Sénégal.

\section{An efficacious bivalent vaccine against Newcastle disease and fowlpox disease, produced in Senegal}

\begin{abstract}
Newcastle disease (ND) and avian pox are major pathologies of village poultry in Senegal. They affect most species of birds and cause considerable economic losses in livestock. Vaccination is the only effective means of prevention; hence the idea of developing a bivalent vaccine to fight these two diseases. The bivalent
\end{abstract}


vaccine produced consists of the Newcastle disease virus (NDV) strain I-2 and a local strain of the avian pox virus (PGPV19). To evaluate the efficacy of bivalent vaccine in the laboratory, vaccine trials were conducted on 21-day-old chickens. The haemagglutination inhibition (HI) technique is used to detect antibodies to NDV, while the immunogenicity of the PGPV19 strain is determined by testing chickens with the wild type S20 strain of avian pox virus. The results obtained are conclusive because the vaccinated chickens produced neutralizing antibodies against ND and displayed immunity when exposed to the S20 strain, unlike the control subjects. However, these results, although encouraging, must be supported by comparative data from field monitoring of village farms.

(C) 2017 International Formulae Group. All rights reserved.

Keywords: Village poultry, Newcastle disease, fowlpox, bivalent vaccine, Senegal.

\section{INTRODUCTION}

La maladie de Newcastle (MN) et la variole aviaire sont les deux pathologies les plus fréquentes en aviculture villageoise au Sénégal (ISRA, 2010). La MN est très contagieuse et meurtrière ; elle est due à un Paramyxovirus aviaire qui est un virus à ARN enveloppé (Alexander, 2003 ; OIE, 2012). La variole aviaire est moins contagieuse que la MN mais peut être transmise par les insectes piqueurs, elle est présente dans le monde entier selon l'Office International des Epizooties (OIE, 2016). La variole aviaire est causée par un Avipoxvirus qui est un virus à ADN (Jarmin et al., 2006 ; Weli et Tryland 2011). La maladie se manifeste sous deux formes: la forme cutanée encore appelée variole sèche et la forme diphtérique appelée variole humide (OIE, 2016). La MN et la variole aviaire sont des maladies redoutables en aviculture villageoise car elles affectent la plupart des oiseaux et elles sont très redoutées par les éleveurs, puisqu'elles occasionnent des pertes économiques considérables. En effet, le taux de mortalité causé par les maladies chez les jeunes sujets au Sénégal est estimé à 43 voire 63\% (Ouedraogo et al., 2015) dont 80\% sont imputables à la MN. Il y a aussi la baisse des performances (baisse de ponte, retards de croissance) causée par ces pathologies, dans un contexte où l'alimentation des sujets est arbitraire et presque toujours carencée (Kuietche et al., 2014). Il n'y a pas de traitement officiellement reconnu pour lutter contre la $\mathrm{MN}$ toutefois certaines plantes et certains minéraux seraient capables d'éliminer le virus de la MN (Ezeibe et al., 2011;
Nyandoro et al., 2014). Par conséquent, la vaccination demeure le seul moyen de contrôle efficace de ces maladies, qui sévissent à l'état endémique au Sénégal, avec des pics épizootiques récurrents. Une stratégie de vaccination de masse de la volaille locale contre la MN est adoptée au Sénégal depuis plusieurs années mais il apparaît de plus en plus des flambées de variole aviaire consécutives à cette immunisation contre la $\mathrm{MN}$. Ceci est à l'origine de l'association vaccinale pour une lutte stratégique et coordonnée contre ces maladies.

La mise au point d'un tel vaccin bivalent est confortée par la ressemblance de leur mode de préparation à base d'œufs embryonnés (OIE, 2012 ; OIE, 2016), des 3 à 4 mois de protection qu'ils confèrent (Alders et Spradbrow, 2001 ; Shil et al., 2007), voire dans leur voie d'administration (Alders et Spradbrow, 2001 ; Ariyoshi et al., 2003). Le vaccin bivalent proposé, est composé en partie de la souche virale du vaccin I-2 qui provient d'une souche avirulente du virus australien de la MN (Alders et Spradbrow, 2001). Le vaccin I-2 est conçu pour une utilisation en aviculture villageoise, en raison de sa thermostabilité et son utilisation facile sous forme de collyre ou orale (eau de boisson). L'autre souche vaccinale qui compose le vaccin bivalent est une souche locale de virus de la variole de pigeon PGPV19, isolée au laboratoire de Hann à Dakar. Par conséquent, dans cette étude, l'efficacité du vaccin bivalent est évaluée au laboratoire suivant deux techniques. Le test de l'inhibition de l'hémagglutination est utilisé pour déceler les 
anticorps induits par le vaccin I-2 chez les sujets qui auront reçu ce vaccin, tandis que, l'efficacité de la souche PGPV19 sera éprouvée avec une souche sauvage de variole, quelques semaines après la vaccination.

\section{MATERIEL ET METHODES}

\section{Le vaccin bivalent}

Il s'agit d'un vaccin à virus vivants, constitué de la souche vaccinale I-2 de la MN (FAO, 2002), utilisée à un titre viral de $10^{7}$ doses infectantes à $50 \%$ pour l'embryon $\left(10^{7}\right.$ $\mathrm{DIE}_{50} /$ dose) et d'une souche locale de variole de pigeon PGPV19, atténuée au laboratoire de Hann en 2015, par passages successifs dans la membrane chorio allantoidienne d'œufs embryonnés de 10-11 jours et ayant un titre de $10^{4,75} \mathrm{DIE}_{50} /$ dose.

\section{La sélection de la souche de variole pour l'épreuve virulente}

Trois souches sauvages provenant de poulets malades de variole aviaire, $\mathrm{S} 02, \mathrm{~S} 20$ et S21 sont testées pour en déterminer la plus virulente. Les poussins sont très sensibles à la variole, aussi, il a fallu tester les souches sur des sujets âgés de 21 jours pour pouvoir déterminer la plus pathogène. Les poulets sont répartis en quatre lots de cinq sujets et chaque lot de volailles est infecté avec une des souches ci-dessus identifiées. Les poulets du quatrième lot (témoins) ne reçoivent que du tampon phosphate (PBS) à pH 7,2. L'inoculation se fait au niveau du barbillon avec $0,5 \mathrm{ml}$ d'inoculum. Il s'en suit un examen quotidien des volailles pendant quinze jours, pour dénombrer le nombre de poulets qui présentent des signes de fébrilité et/ou de lésions cutanées consécutives à l'infection.

\section{La voie d'administration du vaccin}

Dans cette expérience, les voies d'administration choisies sont la voie oculaire et la voie orale, officiellement recommandées pour l'utilisation du vaccin I-2. La vaccination par voie oculaire consiste à introduire une goutte de vaccin dans l'œil du sujet et pour l'administration orale, le groupe d'oiseaux ciblé a été assoiffé pendant 2 heures avant de les abreuver avec $150 \mathrm{ml}$ d'eau contenant 0,3 $\mathrm{g}$ de lait en poudre (tampon) et 10 doses de vaccin. Ce mélange vaccinal est préparé en excès pour permettre à tous les sujets du groupe de boire une dose vaccinale protective.

\section{Le test de l'inhibition de l'hémagglutination (IHA)}

C'est la technique couramment utilisée pour évaluer l'efficacité de la vaccination contre la MN. Le titre en IHA est déterminé par la dilution sérique la plus poussée qui provoque une inhibition complète de 4 unités hémagglutinantes d'antigènes (Alders et Spradbrow, 2001). L'absence d'hémagglutination est visible à l'œil nu puisqu'en inclinant la plaque, une trainée de globules rouges doit couler dans les puits témoins (contenant seulement $0,025 \mathrm{ml}$ d'érythrocytes et $0,05 \mathrm{ml}$ de PBS et tous les puits dans lesquels les érythrocytes coulent de la même façon sont considérés comme présentant une inhibition (OIE, 2012).

\section{L'essai vaccinal}

L'essai vaccinal est réalisé sur un effectif de quarante-neuf $(n=49)$ poussins non sexés d'un jour, élevés pendant 21 jours afin d'éliminer toute trace d'anticorps maternels. Ensuite, ils sont individuellement identifiés puis répartis aléatoirement en quatre groupes physiquement séparés conformément au Tableau 1. Des prélèvements sanguins sont réalisés sur les poulets des groupes 2 et 4 , au temps T0 puis toutes les semaines après la vaccination de ces sujets contre la $\mathrm{MN}$, pour la collecte de sérums nécessaires au test d'IHA. Trois semaines après l'immunisation, les sujets vaccinés avec la souche de variole PGPV19 et le vaccin bivalent I-2/PGPV19 sont mis en contact avec le virus sauvage de la variole aviaire, en leur inoculant $0,5 \mathrm{ml}$ de virus S20 dans le barbillon ou la crête des poulets des groupes 3 et 4 . Les poulets témoins du groupe 1 sont également infectés avec la souche d'épreuve S20. La bande est suivie pendant quinze jours après l'épreuve virulente, avant d'être sacrifiée. 
Tableau 1 : Protocole expérimental d'évaluation de l'efficacité des vaccins I-2; PGPV19 et du vaccin bivalent selon les voies d'administration.

\begin{tabular}{ll}
\hline Groupe 1 & 7 poulets témoins non vaccinées \\
\hline Groupe 2 & 7 poulets vaccinés avec I-2 par voie oculaire \\
& 7 poulets vaccinés avec I-2 par voie orale \\
\hline Groupe 3 & 7 poulets vaccinés avec PGPV19 par voie oculaire \\
& 7 poulets vaccinés avec PGPV19 par voie orale \\
\hline Groupe 4 & 7 poulets vaccinés avec bivalent par voie oculaire \\
& 7 poulets vaccinés avec bivalent par voie orale \\
\hline
\end{tabular}

\section{RESULTATS}

\section{Sélection de la souche d'épreuve de variole}

Pour chaque souche d'épreuve, le nombre de poulets présentant des signes de variole aviaire est déterminé et une représentation graphique est réalisée (Figure 1). La souche $\mathrm{S} 20$ a causé des lésions cutanées chez quatre poulets sur cinq. Les souches $\mathrm{S} 02$ et S21 n'ont donné chacune qu'un seul cas de lésions cutanées sur les cinq poulets (Figure 1). Cependant, aucun poulet du groupe témoin n'a montré de lésions cutanées ni de signes de fébrilité. C'est la souche S20 qui semble la plus virulente.

\section{Test d'inhibition de l'hémagglutination (IHA)}

Pour les poulets vaccinés contre la $\mathrm{MN}$, la réponse immunitaire a été mesurée par un test séro-immunologique. Les tests IHA ont permis de déterminer les titres en anticorps chez les poulets du groupe 2 vaccinés contre la $\mathrm{MN}$ (Figure 2) et ceux du groupe 4 (Figure 3 ). Le vaccin I-2 suscite visiblement la production d'anticorps et le titrage de ces anticorps permet de voir qu'il y a une évolution rapide du titre, avec un pic vers le $14^{\text {ème }}$ jour.

\section{Essai vaccinal}

Les poulets du groupe 3 (Figure 4) et du groupe 4 (Figure 5) n'ont présenté aucun cas de variole aviaire après avoir été éprouvés avec la souche sauvage S20, alors que parmi le groupe témoin, cinq poulets sur sept ont développé des lésions cutanées.

\section{DISCUSSION}

Cette étude a permis de confirmer l'adéquation des souches locales de variole aviaire pour la fabrication de vaccins comme l'avaient démontré d'autres équipes de recherche (Mohamed et al., 2004 ; Islam et al., 2008). La souche virale de la variole de pigeon est choisie comme souche vaccinale puisqu'elle est naturellement atténuée (Shil et al., 2007 ; Masola et al., 2016), contrairement aux souches de variole provenant d'autres espèces de volatiles (Haller et al., 2014). Plus précisément, la souche PGPV19, présente l'avantage de ne pas être associé au virus de la réticuloendothéliose (REV). Le REV est un contaminant naturel du virus de la variole aviaire (Tadese et Reed, 2003; Witter et Fadly, 2003 ; Awad et al., 2010; Biswas et al., 2011 ; Masola et al., 2014). Cependant, ce phénomène d'intégration du génome du virus de la REV dans le virus de la variole aviaire, bien que largement répandu dans le monde (Kim et Tripathy, 2001; Mzula et al., 2014), n'est au demeurant, ni un phénomène exhaustif, ni systématique (Davidson et al, 2008). Toutefois, la présence du virus de la REV dans le virus de la variole aviaire joue un rôle très important dans la virulence des souches de virus de variole en circulation (Wang 2002 ; Singh et al., 2003). 


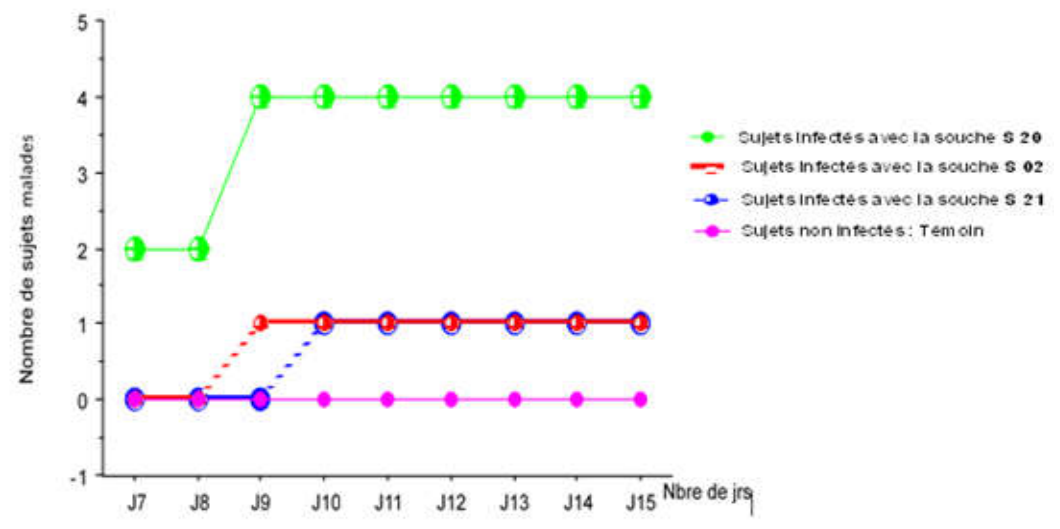

Figure 1: Courbes d'évolution du nombre de poulets infectés par le virus de la variole aviaire selon la virulence de la souche d'infection.
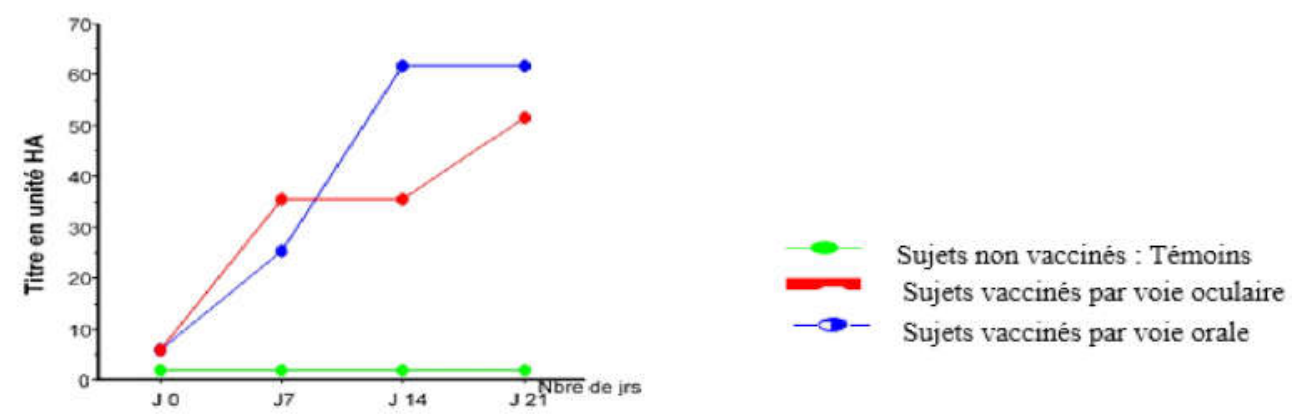

Figure 2: Courbe de réponse en anticorps des poulets vaccinés avec le vaccin I-2 suivant les deux voies d'administration.

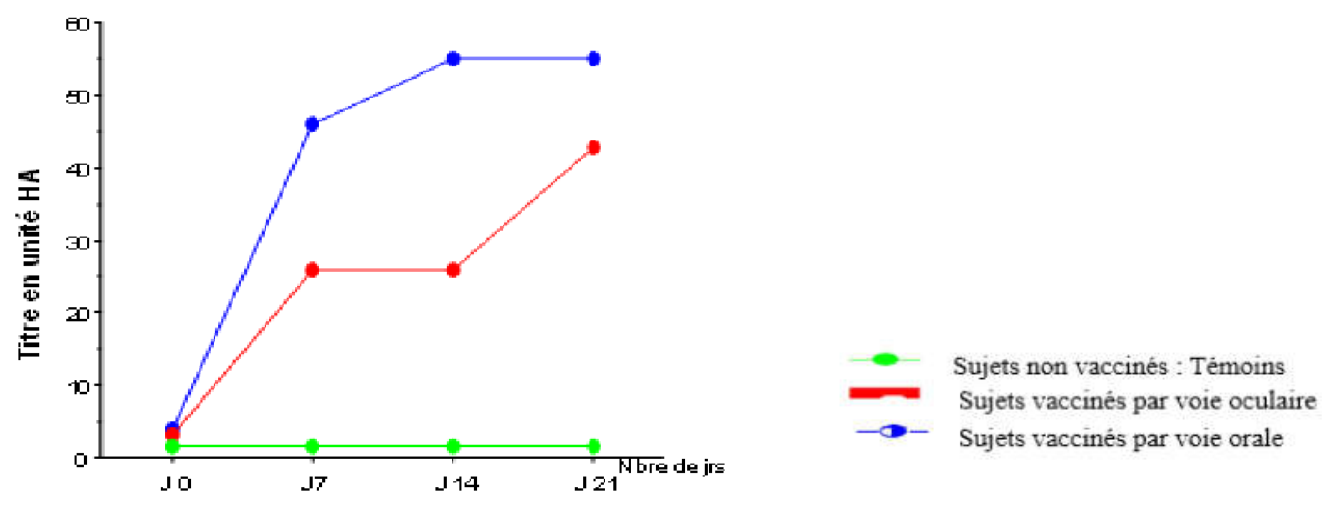

Figure 3: Courbe de réponse en anticorps des poulets vaccinés avec le vaccin bivalent I-2/PGPV19 suivant les deux voies d'administration. 


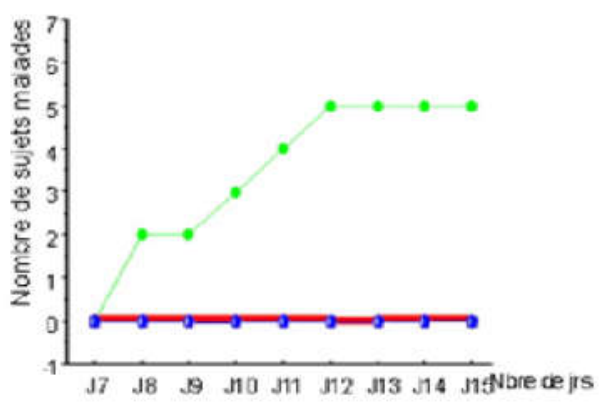

Sujets non vaccinés : Témoins

Sujets vaccinés par voie oculaire

Sujets vaccinés par voie orale

Figure 4 : Courbe d'évolution des poulets vaccinés avec le vaccin PGPV19 puis infectés avec la souche de variole S20.

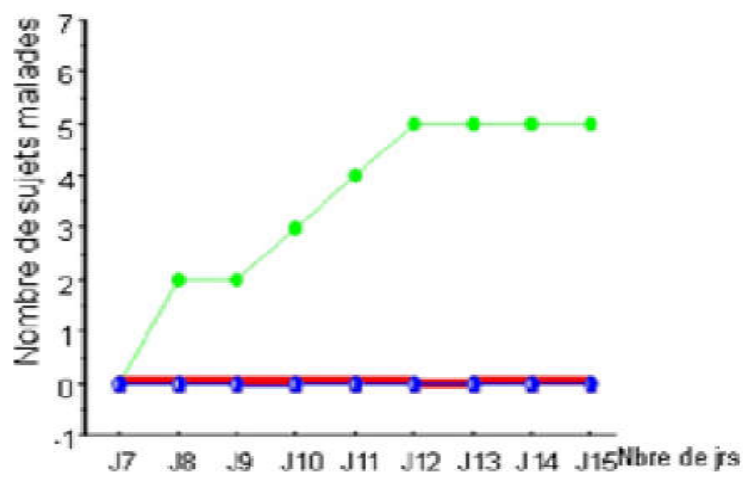

Figure 5: Courbe d'évolution des poulets vaccinés avec le vaccin bivalent, I-2/PGPV19 puis infectés avec la souche de variole S20.

Une analyse par PCR préalable avait révélé que les trois souches d'épreuve S02, S20 et S21 avaient intégré le virus de la REV dans leur génome. Cela laissait présager une certaine virulence de ces souches. Mais, seule la souche S20 s'est avérée fortement infectieuse, elle a été retenue comme souche d'épreuve. En fait, le groupe des REV est composé de virus défectueux oncogènes et de virus non défectueux qui sont faiblement infectieux (Barbosa, 2006). Les virus de la variole pouvant intégrer l'une ou l'autre de ces formes de REV dans leur génome, sont à l'origine de la grande variabilité génétique des souches de variole en circulation (El Mahdy et al., 2014 ; El Sebelgy et al., 2014) mais aussi de la variation de virulence observée (Garcia et al., 2003). En conséquence, les résultats obtenus avec les différentes souches S02, S20 et $\mathrm{S} 21$, confirment bien les observations de ces différents auteurs.

Par respect de la réglementation relative à la protection des animaux utilisés à des fins scientifiques et conformément au principe des 3R (réduire, remplacer et raffiner) (Russel, 2005), le test sérologique 
d'IHA est utilisé pour évaluer l'efficacité du vaccin I-2 à la place de l'épreuve virulente. Ainsi, il a permis de voir que la souche I-2 conférait une bonne immunité puisque les poulets des groupes 2 et 4 ont développé des anticorps sériques contre la $\mathrm{MN}$ alors que les témoins n'ont pas fait de séroconversion. L'efficacité du vaccin I-2 a été largement démontrée par de nombreux auteurs (Illango et al., 2005; Komba et al., 2012), aussi il n'a pas été envisagé de la reprendre dans cette expérience. Par contre, pour la souche PGPV19, il a fallu réaliser une expérimentation animale puisqu'il s'agit d'une souche locale de terrain et il s'est avéré nécessaire d'en évaluer l'innocuité et la virulence conformément aux normes internationales pour l'harmonisation de l'enregistrement des médicaments vétérinaires (VICH) (Ph Eur, 2013). Les sujets des groupes 3 et 4 préalablement vaccinés ont affiché une certaine immunité lorsqu'ils sont infectés avec la souche S20, comparativement aux témoins du groupe 1 . Les résultats obtenus avec la souche vaccinale PGPV19 sont conformes à ceux de Wang et al. (2002). Les critères fixés pour le choix de la souche PGPV19 sont similaires à ceux de Boyle (2003) qui ont pu identifier, tester et proposer une souche vaccinale de variole aviaire conforme aux exigences de la pharmacopée européenne (Ph Eur, 2001), en termes d'innocuité, d'efficacité et de pureté de la souche (absence de REV). Le test d'IHA et l'épreuve virulente montrent que le vaccin bivalent se révèle efficace aussi bien pour une protection contre la $\mathrm{MN}$ que contre la variole aviaire. Il ressort de cette étude qu'une immunisation par voie orale ou oculaire $d u$ vaccin bivalent est possible même si la transfixion de la membrane alaire demeure la voie d'administration recommandée du vaccin contre la variole aviaire (OIE, 2016). Ce vaccin bivalent est destiné à l'aviculture familiale, cela justifie le choix d'une administration plus facile comme la voie orale ou oculaire compte tenu du faible effectif des élevages villageois. Cette expérience a également montré que pour les deux voies d'administration testées, il y a une réponse immunitaire qui est comparable à celle des vaccins monovalents administrés séparément. Cela confirme l'absence d'interférence entre les activités des deux virus et vient corroborer des travaux antérieurs (Sharma et al., 2002) sur la compatibilité entre la souche de variole aviaire et celle de la MN.

\section{Conclusion}

La combinaison vaccinale exige au préalable que les préparations constitutives soient compatibles et que les voies d'administration soient harmonisées. L'efficacité d'un tel vaccin n'est avérée que si son administration confère pour chaque valence une protection équivalente à celle de l'administration séparée des vaccins. L'association du vaccin I-2 avec une autre souche vaccinale est certainement la première expérience tentée en Afrique et les résultats obtenus montrent que le vaccin bivalent produit est efficace et présente une certaine innocuité. Il serait cependant utile de conforter ces résultats par des données comparatives issues de suivis d'élevages en milieu réel. De nos jours, de nombreux vaccins aviaires sont combinés pour des raisons pratiques et économiques. Il s'agit pour l'essentiel de vaccins recombinants et le génome de la variole aviaire est très souvent utilisé comme vecteur. En effet, compte tenu de la taille de son $\mathrm{ADN}$, le virus de la variole aviaire se 
prête aisément à l'insertion de gènes étrangers. C'est le cas des vaccins TROVAC du laboratoire Mérial et le Vectomune du laboratoire Ceva qui sont constitués du génome de la variole aviaire, dans lequel sont insérés des gènes $\mathrm{N}, \mathrm{H}$ et $\mathrm{F}$ du virus de la MN. De tels vaccins sont aussi des bivalents qui permettent de lutter simultanément contre la MN et la variole aviaire. Le champ des vaccins vectorisés offre ainsi beaucoup de perspectives surtout pour les vaccins aviaires.

\section{CONFLIT D'INTERETS}

Les auteurs déclarent qu'ils n'ont aucun conflit d'intérêts.

\section{CONTRIBUTIONS DES AUTEURS}

NFTN a coordonné et planifié l'expérimentation, assuré la revue de littérature et rédigé l'article, EHT a fourni la littérature grise et a relu le manuscrit. MA et AS ont suivi l'expérimentation et ont fait des tests sérologiques. MA a tracé les graphes, AS et MD ont analysé les souches par PCR. FTL a fourni des souches de terrain, ANN a fait des tests sérologiques, $\mathrm{MML}$ et $\mathrm{AD}$ ont joué le rôle d'encadreurs.

\section{REMERCIEMENTS}

Nous remercions tous les techniciens et les manœuvres de l'Unité de production de vaccins et $\mathrm{du}$ Laboratoire national de l'élevage et de recherches vétérinaires de l'Institut Sénégalais de Recherches Agricoles (ISRA) qui n'ont ménagé aucun effort pour le bon déroulement de ces travaux.

\section{REFERENCES}

Alders R, Spradbrow P. 2001. Controlling Newcastle Disease in Village Chickens. ACIAR Monograph No. 82, Australian
Centre for International Agriculture Research: Canberra, Australia; 112.

Alexander DJ. 2003. Newcastle disease, other avian paramyxoviruses, and pneumovirus infections. In Diseases of Poultry, YM Saif (ed). Iowas State University Press: Ames, IA, USA: 6387.

Ariyoshi R, Takase K, Matsuura Y, Deguchi K, Ginnaga A, Fujikawa H. 2003. Vaccination against Fowlpox virus via drinking water. J. Vet. Med. Sci., 65(10): 1127-1130.

Awad AM, Abd El-Hamid HS, Abou Rawash AA, Ibrahim HH. 2010. Detection of reticuloendotheliosis virus as a contaminant of fowlpox vaccines. Poultry Science, 89: 2389-2395.

Barbosa TMC. 2006. Pathogenicity, Tansmission and Molecular characterization of Reticuloendotheliosis virus strain APC-566. MSc Thesis University of Georgia.

Biswas SK, Jana C, Chand K, Rehman W, Mondal B. 2011. Detection of fowlpox virus integrated with reticuloendotheliosis virus sequences from an outbreak in backyard chickens. India.Veterinaria Italiana 47(2): 147153.

Boyle DB. 2003. New Fowl Pox Vaccine Evaluation - Evaluation of fowlpox (FPV) strains free of reticuloendotheliosis virus (REV) as vaccines for use in Australian poultry flocks. RIRDC Project No CSA16 A final report.

Davidson I, Shkoda I, Perk S. 2008. Integration of the reticuloendotheliosis virus envelope gene into the poultry fowlpox virus genome is not universal. 
Journal of General Virology, 89: 24562460.

El-Mahdy SS, Awaad MHH, Soliman YA. 2014. Molecular identification of local field isolated fowlpox virus strain from Giza governorate of Egypt, Veterinary World. 7(2): 66-71.

El-Sebelgy MM, Ahmed BM, Ata NS, Hussein HA. 2014. Molecular detection and characterization of reticuloendotheliosis virus in broiler breeder chickens with visceral tumors in Egypt. International Journal of Veterinary Science and Medicine, 2: 2126.

Ezeibe MCO, Ijabo O, Uzopuo C, Okoroafor ON, Eze JI, Mbuko IJ, Sanda ME, Animoke PC, Ngene AA. 2011. Effects of Aluminium-Magnesium Silicate on Newcastle Disease Virus and on Recovery of Infected Chicks. International Journal of Biological and Chemical Sciences, 5: 825829. http://dx.doi.org/10.4314/ijbcs.v5i2. 72160

Garcia M, Narang N, Reed WM, Fadly AM. 2003. Molecular characterization of reticuloendotheliosis virus insertions in the genome of field and vaccine strains of fowlpox virus. Avian Dis., 47: 343354.

Haller SL, Peng C, McFadden G, Rothenburg S. 2014. Poxviruses and the evolution of host range and virulence. Infection, genetics and evolution. Journal of Molecular Epidemiology and Evolutionary Genetics in Infectious Diseases, 15-40. http://doi.org/10.1016/ j.meegid.2013.10.014

Illango J, Olaho-Mukani W, Mukiibi-Muka G, Abila PP, Etoori A. 2005.
Immunogenicity of a locally produced Newcastle disease I-2 thermostable vaccine in chickens in Uganda. Trop Anim Health Prod, 37(1):25-31.

Institut Sénégalais de Recherches Agricoles (ISRA). 2010. Rapport sur les résultats des enquêtes sur les principales pathologies en aviculture familiale au Sénégal. PPAAO/WAAPP/SEN/Phase 1 projet

FNRAA 09/AP07SS011007/G2."Amélioration des conditions d'exploitation de l'aviculture traditionnelle en milieu rural par une réduction de l'incidence de la maladie de Newcastle et des parasitoses"

Islam MR, Khan MSR, Islam MA, Kayesh MEH, Karim MR, Gani MO, Kabir A. 2008. Comparative efficacy of imported Fowlpox virus vaccine with locally produced one in backyard chicks. Bangl. J. Vet. Med., 6(1): 23-26.

Jarmin S, Manvell R, Gough RE, Laidlaw SM, Skinner MA. 2006. Avipoxvirus phylogenetics: identification of a PCR length polymorphism that discriminates between the two major clades. Journal of General Virology, 87: 2191-2201.

Kim TJ, Tripathy DN. 2001. Reticuloendotheliosis virus integration in the fowlpox virus genome: not a recent event. Avian Dis., 45: 663-669.

Komba EVG, Mbyuzi AO, Mulangila RCT. 2012. Adoption of I-2 Vaccine in Immunization of Village Chickens against Newcastle Disease Virus in Southern Tanzania: Immune Status of Farmer Vaccinated Birds. Journal of Agricultural Science. 4(4).

Kuietche HM, Kana JR, Defang HF, Tadondjou CD, Yemdjie DDM, Teguia, A. 2014. Effect of dietary energy level 
on growth performance and morphometric parameters of local barred chickens at the starter phase. International Journal of Biological and Chemical Sciences, 8(3): 882890. http://ajol.info/index.php/ijbcs

Masola SN, Mzula A, Kasanga CJ, Wambura PN. 2014. Integration of Reticuloendotheliosis Virus in Most of Tanzanian FowlPox Virus Isolates is not attributed to Imported Commercial FowlPox Vaccines. British Biotechnology Journal, 4(6): 659-669.

Masola SN, Mzula A, Kasanga CJ, Wambura PN. 2016. Evaluation of Virulence of Tanzanian Strains of Fowlpox and Pigeonpox Viruses in Chickens. British Biotechnology Journal, 10(1): 1-10. DOI: 10.9734/BBJ/2016/20150.

Mohamed Elamin UM, Mohamed Elhossain A, Mohamed Nour TA, Saeed Ali A. 2004. Performance of a locally produced fowlpox vaccine under laboratory and field conditions in the Sudan. J. Sc. Tech, $5(1)$.

Mzula A, Masola SN, Kasanga CJ, Wambura PN. 2014. Existence of variant strains Fowlpox virus integrated with Reticuloendotheliosis virus in its genome in field isolates in Tanzania. Trop Anim Health Prod. DOI 10.1007/s11250-0140552-2

Nyandoro SS, Nkunka MHH, Cosam JC, Msoffe PLM. 2014. In ovo antiviral potency of the leaf constituents of Tanzanian Toussaintia species against Infectious Bursal Disease Virus and Newcastle Disease Virus. Int. J. Biol. Chem. Sci., 8(3): 1308-1318 DOI: http://dx.doi.org/10.4314/ijbcs.v8i3.43
Office International des épizooties (O.I.E) 2012 Terrestrial Manual; Newcastle Disease (infection with Newcastle disease virus), 555-574.

Office International des épizooties (O.I.E). 2016. Terrestrial Manual. Fowlpox Ed. OIE: Paris; 8.

Organisation des Nations Unies pour l'Alimentation et l'Agriculture (FAO). 2002. TCP, SEN 0065/D.

Ouedraogo B, Bale B, Zoundi SJ, Sawadogo L. 2015. Caractéristiques de l'aviculture villageoise et influence des techniques d'amélioration sur ses performances zootechniques dans la province du Sourou, région Nord-Ouest Burkinabè. Int. J. Biol. Chem. Sci., 9(3): $\quad$ 1528-1543. DOI: http://dx.doi.org/10.4314/ijbcs.v9i3.34

Pharmacopée Européenne (Ph. Eur.). 2001. Pharmacopée Européenne, $4^{\mathrm{eme}}$ edn.

Russell WMS. 2005. The Three Rs: Past, Present and Future, Animal Welfare 14, 279-286.

Sharma JM, Zhang Y, Jensen D, Rautenschlein S, Yeh HY. 2002. Field trial in commercial broilers with a multivalent in ovo vaccine comprising a mixture of live viral vaccines against marek's disease infectious bursal disease, newcastle disease, and fowl pox. Avian Diseases, 46(3): 613-622. Doi: http://dx.doi.org/10.1637/0005-2086 (2002)046

Shil NK, Rahman MS, Hossain MT, Islam MT, Rahman MM, Islam KBMS, Cha SY, Jang HK, Song HJ. 2007. Immune response and efficacy of pigeon pox virus vaccine and fowlpox virus vaccine in chickens. Kor. J. Vet. Serv., 30(4): 497503. 
Singh P, Schnitzlein WM, Tripathy DN. 2003.

Reticuloendotheliosis Virus Sequences within the Genomes of Field Strains of Fowlpox Virus Display Variability. Journal of Virology, 77: 5855-5862.

Tadese T, Reed WM. 2003. Detection of specific reticuloendotheliosis virus sequence and proteinfrom REVintegrated fowlpox virus strains. Journal of Virological Methods, 110(1): 99-104.

Wang J, Meers J, Spradbrow P, Robinson WF. 2002. Evaluation of immune effects of fowlpox vaccine strains and field isolates Veterinary Microbiology, 116(1): 106-119.
Wang J. 2002. Comparative virulence of Australian Fowlpox vaccine strains and field isolates $\mathrm{PhD}$ Thesis, School of Veterinary Science, The University of Queensland.

Weli SC, Tryland M. 2011. Avipoxviruses: infection biology and their use as vaccine vectors, Virology Journal, 8: 49.

Witter RL, Fadly AL. 2003. Reticuloendotheliosis. In Diseases of Poultry ( $11^{\mathrm{e}}$ edn), Saif YM, Barnes J, Glisson J, Macdougal L, Swayne D (eds). Iowa State Press: Ames; 517-535. 